\title{
25 Research Soure \\ The Effects of Hydrogen-Rich Water on Skin Wound Healing in Dogs
}

Dong-Dong Qi

Northeast Agricultural University

Ting Wang

Northeast Agricultural University

Muhammad Abid Hayat

Northeast Agricultural University

Tao Liu

Northeast Agricultural University

JianTao Zhang ( $\sim$ zhangjiantao@neau.edu.cn )

Northeast Agricultural University

\section{Research Article}

Keywords: antioxidant, dogs, hydrogen-rich water, wound healing.

Posted Date: May 26th, 2021

DOl: https://doi.org/10.21203/rs.3.rs-514630/v1

License: (c) (1) This work is licensed under a Creative Commons Attribution 4.0 International License. Read Full License 


\section{Abstract}

Background: Hydrogen-rich water (HRW) has been shown to be one of underlying therapeutic strategies regarding wound treatments. This study explored the effects of drinking HRW on skin wound healing in dogs. Eight circular wounds were analyzed in each dog. The experimental group was treated with HRW twice daily, while the control group was provided with distilled water (DW). The wound tissues of dogs examined histopathologically. The fibroblasts, inflammatory cell infiltration, the average number of new blood vessels, and the level of malondialdehyde (MDA) and superoxide dismutase (SOD) activity in the skin homogenate of the wound was measured using the corresponding kits. The expression of Nrf-2, HO1, NQO-1, VEGF, and PDGF were measured by real-time fluorescence quantitative method.

Results: We observed that HRW wound had significant average healing rate and faster average healing time. Histopathological results showed that the average number of blood vessels and the average thickness of epidermis were significantly different from the DW group. The MDA levels were higher in the DW group than in the HRW group but the SOD levels were higher in the HRW group than in the DW group. The results of qRT-PCR showed that the expression of each gene was significantly different between the two groups.

Conclusions: The results showed that HRW treatment could promote skin wound healing in dogs, accelerate wound epithelization, reduce inflammatory reaction, stimulate the expression of cytokines related to wound healing, and shorten wound healing time.

\section{Background}

As the largest organ of animals, the skin plays a critical role in preventing animals from pathogenic microorganisms and wounds [1]. Briefly, the skin wounds caused by diverse elements usually occur in clinical veterinary setting. The recovery of severe wounds associated with numerous factors, such as therapy duration and treatment strategies might lead to various therapeutic effects affecting the time and effects of wound healing [2]. wound healing involves a series of mechanisms, such as inflammatory response, oxidative stress, etc., which play vital roles in wound pathophysiology [3]. Numerous therapeutic approaches have been applied in clinical treatments, including photodynamic therapy, farinfrared ray therapy, and adipose tissue extraction therapy [4-6].

Hydrogen gas has the lowest molecular weight, along with a strong antioxidant capacity to remove excessive reactive oxygen species (oxygen-free radicals) from the body [7]. Clinical studies have shown that hydrogen therapy has therapeutic potential for the treatment of rheumatoid arthritis, diabetes, Parkinson's disease, and metabolic syndrome $[8,9]$. Tomohiro et al. found that hydrogen molecules could activate the NF-E2-related factor (Nrf-2) antioxidant defense pathway and ameliorate hyperoxic lung injury via the induction of Nrf-2 dependent genes, such as HO-1 [10]. Several studies have suggested that the inhalation of hydrogen-containing gas constitutes a novel guideline for practical clinical care, focusing on the treatment of skin wounds [11]. 
Hydrogen gas is a flammable and explosive gas, which is dangerous and difficult to store; however, hydrogen gas-saturated water, which is known as hydrogen-rich water (HRW), is economically safe and easy to absorb, and is frequently used in medicine [10]. HRW has been reported to exhibit antioxidant and anti-inflammatory effects, reducing the levels of pro-inflammatory cytokines, effectively protecting against organ damage [12]. Hydroxyl radical and peroxynitrite (ONOO-) ion have strong oxidation toxicity, and the potential to cause lipid peroxidation, DNA damage, and protein peroxidation, resulting in cell damage [13]. However, hydrogen dissolved in water can react with them, reducing the consequent damage. The four methods commonly used to infuse $\mathrm{H} 2$ gas into water involve high pressure, electrolyzing water to producing $\mathrm{H} 2$, and reacting magnesium metal or its hydride with water to produce HRW [14].

Previous studies on animal testing have used both oral ingestion of HRW and injection of hydrogen-rich saline (HRS) as major routes of administration of HRW [15]. Studies have shown that HRS can scavenge hydrogen peroxide and reactive oxygen species (ROS) $[16,17]$ by upregulating the expression of heme oxygenase (HO-1) as well as the reduction of quinine (NQO-1) [18]. Additionally, recent studies have demonstrated the antifatigue effects of HRW in animals and humans [19]. HRW also induces antiapoptotic effects in myocardial cells [20] and inhibits the production of a, $\beta$-dicarbonyl compounds and ROS in the kidneys of rats [21]. The treatment principle of HRW is mainly reflected by its biological roles in anti-inflammation and antioxidation. Another study suggested that the use of HRW could enhance the rate of palatal wound recovery [22]. Thus, HRW has been shown to be one of underlying therapeutic strategies regarding wound treatments. Although HRW has been proven to accelerate the recovery of skin wounds [11], there is little information available on the use of HRW in the trauma associated with canine skin.

Here we aimed to explore whether HRW could play an essential role in the recovery of skin wounds in a canine model. The specific objectives were (1) to compare the effect of the healing of open wounds in dogs treated with HRW and the ones treated with distilled water (DW); (2) to compare the level of the oxidative stress and inflammatory indicators; (3) to explore whether or not HRW had therapeutic value in the recovery of canine skin wound. We hypothesized that HRW promoted angiogenesis and elevated tissue antioxidant gene expression to promote wound healing and accelerate the rate of wound healing, making it a safe and effective clinical treatment.

\section{Materials And Methods}

\section{Dogs}

Twelve healthy male beagle dogs (age: 2-4 y; weight: 9.0-11.3 kg) were from Breeding Base of Harbin and housed in a standard environment and provided free access to standard dog food and water. Food and water were withdrawn $6 \mathrm{~h}$ prior to anesthesia. The Laboratory Animal Welfare and Ethics Committee of Northeast Agricultural University (\#NEAU-2019-04-0245-13) approved the experimental design and animal surgery procedures in this study. The study was carried out in compliance with the ARRIVE 
guidelines. All methods were handled in accordance with good animal practices required by the Animal Ethics Procedures and Guidelines of the People's Republic of China. Make every effort to minimize animal suffering and reduce the number of animals used. No deaths were reported in this study; both mental status and diet of the dogs were normal. No animals were killed and all dogs were healthy and adopted by animal protection organization after the experiment was finished.

\section{Surgical Operation}

Dogs were randomly divided into control and experimental groups; each group contained six dogs. The hair was clipped on the dorsolateral trunk on day 0 . Cephalic intravenous catheter was placed, and the dogs were anesthetized using propofol $(0.65 \mathrm{mg} / \mathrm{kg})$, followed by the inhalation of $3.5 \%$ isoflurane via endotracheal intubation, and the physiological state of the experimental dogs was monitored in real time during the operation.

Four circular wounds with a diameter of $15 \mathrm{~mm}$ were created on each side of the midline of the shaved dorsum using a dermal biopsy punch. Each wound was $20 \mathrm{~mm}$ from the midline of the dorsum, and the adjacent wounds were $25 \mathrm{~mm}$ from each other. From the head to the tail side, and from left to right, the wounds were labeled as A1, A2, A3, A4, B1, B2, B3, B4 (Fig. 1). Post-operative pain was managed with meloxicam anesthetic ( $0.1 \mathrm{mg} / \mathrm{kg}$, subcutaneously). Each dog was kept in single cage, wearing Elizabethan collars. The wound area was disinfected daily using iodophor. Hydrogen gas $(99.99 \%, \mathrm{v} / \mathrm{v})$ produced from a hydrogen gas generator (QL-500; Saikesaisi Hydrogen Energy Co., Ltd., Shandong, China) was bubbled into $400 \mathrm{~mL}$ of distilled water at the rate of $500 \mathrm{~mL} / \mathrm{min}$ for $10 \mathrm{~min}$ to achieve saturation. All dogs had free access to standard food after postoperative fasting; the control group was provided DW $(400 \mathrm{~mL})$ twice a day and the experimental group was provided saturated HRW $(400 \mathrm{~mL})$ twice a day. Each dog had only one hour at a time to drink to keep the concentration of HRW constant.

Skin wound samples were collected at 7, 14, 21, and 28 day after surgical operation, intubation, analgosedation, and monitoring; the surgical procedures were identical to the skin wound model. The healthy skin around $1 \mathrm{~cm}$ of the wound was removed when skin wound sampling was performed. The skin wound at $A 1$ and $B 1$ was sampled on day $7 ; A 2$ and $B 2$ were sampled on day $14, A 3$ and B3 were sampled on day $21, A 4$ and $B 4$ were sampled on day 28 . Following sampling, the wound was closed using a suture and sterilized with iodophor. $A 1, A 2, A 3$, and $A 4$ samples were fixed in $4 \%$ paraformaldehyde (20g paraformaldehyde powder dissolved in $500 \mathrm{~mL}$ PBS buffer, followed by constant stirring using a magnetic stirrer. $\mathrm{NaOH}$ was used to adjust the solution $\mathrm{pH}$.). $\mathrm{B} 1, \mathrm{~B} 2$, $\mathrm{B} 3$, and $\mathrm{B} 4$ samples were stored in a refrigerator at $-80^{\circ} \mathrm{C}$.

\section{Examination of the skin wound}

The changes in the wounds in the HRW and the DW groups were observed on days 7, 14, 21, and 28 after the operation for bleeding, inflammatory exudate traits, wound healing, wound infection, etc. The wounds were photographed on days $3,7,14$, and 21 , and wound are was quantified using the Image $\mathrm{J}$ software.

Wound healing rate $=(S 0-S n) \div S 0 \times 100 \%$. 
where Sn, S0 (the wound area at 0 day).

Dog skin wound healing was measured until granulation growth for $100 \%$ of the wound; the time of this process was the time of wound healing.

Paraffin sections were prepared for histopathological examination. H\&E staining was used for evaluating epithelial thickness and neovascularization and Masson staining for collagen fiber. Briefly, five sections were chosen from each dog, and ten visual fields were randomly selected from different areas in each section. The evaluation of section was performed by two independent and blinded observers.

Oxidative stress was assessed by detecting malondialdehyde (MDA) and super oxide dismutase (SOD) levels in skin tissues following the instructions on the detection kits (Jiancheng Biotech Nanjing).

Total RNA was isolated from skin tissue using TRIzol Reagent (Invitrogen). The RNA was reverse transcribed using Super Script First Strand cDNA System (Invitrogen), and qRT-PCR was performed using the Light Cycler 480 Real-Time PCR System (Roche Diagnostics, Germany). Table 1 provides the list of primer sequences.

Table 1

Primer sequence

\begin{tabular}{|llll|}
\hline Gene & Gene sequence numbers & Primer sequence (5'to3') & Size (bp) \\
\hline GAPDH & NM_008084.2 & Forward:TCATGAGGCCCTCCACGAT & 157 \\
& & Reverse:GATGGGCGTGAACCATGAG & \\
NRF-2 & NM_009925.4 & Forward:CCCATCGGAAACCAGTGCAT & 150 \\
& & Reverse: CATCTACGAACGGGAATGTCTCTG & \\
HO-1 & Gl: 678541 & Forward: GCCAAGACTGCCTTCCTGCTG & 113 \\
& & Reverse: ACCCGTTGTCGTAGCCCTGAG & \\
NQ01 & NM_008607.2 & Forward: ACCTGTACGCCATGAACTTCAACC & 149 \\
& & Reverse: CTTCTGCTCGGCCACGATGTC & \\
VEGF & NM_008607.2 & Forward:GCCTTGCCTTGCTGCTCTACC & 84 \\
& & Reverse: CTTCGTGGGGTTTGTGCTCTCC & \\
PDGF & NM_008607.2 & Forward:CCTGGCGTGCAAGTGTGAGAC & 112 \\
& & Reverse: CCGAATGGTCACCCGAGTTTGG & \\
\hline
\end{tabular}

\section{Statistical Analysis}

All data were statistically analyzed using independent-sample t-test using SPSS 22.0 statistical software. All results are expressed as mean $\pm \mathrm{SD} ; P>0.05$ indicates no significant difference, $P<0.05$ indicates a 
significant difference, and $P<0.01$ indicates that the difference was extremely significant.

\section{Results}

\section{Wound Healing}

\section{Clinical Wound Evaluation}

Figure 2 shows the presence of a significant difference in skin wound area between the HRW and DW groups between days 3 and 21. On day 3, the skin wound showed obvious hemorrhage, edema, and inflammatory reaction in the DW group and was not obvious in the HRW group. On day 7, there were abundant granulation tissue, newborn epithelia, and wound surfaces were humid and glossy in the HRW group, whereas the DW group had fewer granulation tissues and newborn epithelia, and wound surfaces were dried and matte, with large necrotic tissues and with inflammatory exudate. On day 14, skin tissue defects were filled with granulation tissue in all dogs; the HRW group showed better re-epithelialization than the DW group with the appearance of scar tissue and hair growth. On day 21, wounds were almost fully re-epithelialized in the HRW group. On day 28 , it showed complete wound closure and healing in both HRW and DW groups.

\section{Wound Healing Rate and Average Healing Time}

During the experimental period, the rate of skin wound healing in the DW and HRW groups increased gradually until complete healing was achieved (Fig. $3 \mathrm{~A})$. On day 3 , the HRW group $(17.57 \pm 0.55 \%)$ had a significantly higher rate of skin wound healing compared with that of the DW group $(1.59 \pm 0.55 \%)(P<$ $0.05)$. On day 14 , the HRW group $(46.82 \pm 4.29 \%)$ had an extremely significantly higher rate of skin wound healing compared with that of the DW group $(16.44 \pm 6.43 \%)(P<0.01)$. The average duration of wound healing (Fig. 3B) was ( $19.00 \pm 0.36 \mathrm{~d})$ in the HRW group, which was significantly different than the DW group $(22.00 \pm 1.10 \mathrm{~d})(P<0.05)$.

\section{Histopathology}

\section{Wound length}

The results from histopathological analysis of skin showed that on day 7, the wound area was still large and the surface of wound was covered with the granulation tissue in DW and HRW groups. On day 14, the skin wound area in the HRW group was significantly smaller and showed better re-epithelialization than the DW group $(P<0.05)$.

\section{Re-epithelialization}

The formation of neoepithelium was observed along the wound surface margin in the HRW group, initiating keratinocyte differentiation, epidermal cell differentiation, epidermis development, skin 
development, and keratinization. On day 14, undifferentiated keratinocytes were found in the DW group. On day 21, the HRW group showed marked reduction in epithelial flattening, gland formation, and epithelium thickness, whereas the DW groups showed irregularly thick epithelium (Fig. 5A). Reepithelialization were finished in the HRW group by day 28 , with obvious appearance of connective tissue, which indicated the appearance of scar tissue. On day 14 , the average epidermal thickness was significantly lower in the HRW group $(153.42 \pm 31.72 \mu \mathrm{m})$ than the DW group $(193.28 \pm 45.80 \mu \mathrm{m})(P<$ 0.05 ; Fig. $5 B$ ). On day 21 , the average epidermal thickness was extremely significantly lower in the HRW group $(54.16 \pm 2.53 \mu \mathrm{m})$ compared with the DW group $(123.54 \pm 46.14 \mu \mathrm{m})(P<0.01)$. On day 28, all skin wounds in the canines had been healed and there was no significant difference between the HRW and DW groups regarding the average epidermal thickness.

\section{Neovascularization}

Both HRW and DW groups showed the highest number of neovascular structures on day 7 , which gradually decreased with time (Fig. $5 A)$. On day 7 , compared with the DW group ( $8.60 \pm 2.97)$, there was a significantly higher number of neovascular structures in the HRW group $(17.60 \pm 1.14)(P<0.01)$. On day 14 , there was no extremely significant difference between the two groups. On day 21 , compared with the DW group ( $3.20 \pm 0.81)$, there was an extremely significant difference in the number of neovascular structures in the HRW group $(1.20 \pm 0.84)(P<0.01$; Fig. 5 C).

\section{Collagen Fiber}

The collagen fibers were stained blue, and the muscle fibers were stained red after Masson's trichrome staining. The results of the pathological section showed an increase in the collagen fibers with time both in the DW and HRW groups (Fig. 6). The collagen fibers in the HRW group were configured regularly between days 7 and 21 and were similar to healthy skin until day 28. The DW group showed staggered and irregular collagen fibers between days 7 and 21 and were fewer than those in the HRW group. On day 28 , there was a dense deposition of irregular collagen fibers in the DW group.

\section{Oxidative Stress Detection}

During the experimental period, the level of MDA in skin tissues (Fig. 7A) first increased (from day 7 to 14) and then decreased (from day 14 to 28 ) in both DW and HRW groups. On day 7, compared with the levels of MDA in the skin tissue in the DW group $(31.05 \pm 4.51 \mathrm{U} / \mathrm{mg})$, the same in the HRW group $(17.68 \pm 2.48$ $\mathrm{U} / \mathrm{mg})$ was extremely significantly lower $(P<0.01)$. On day 14 , the MDA levels in the DW group $(41.40 \pm$ $2.74 \mathrm{U} / \mathrm{mg})$ were extremely significantly higher than that in the HRW group $(17.47 \pm 2.82 \mathrm{U} / \mathrm{mg})(P<0.01)$. Between days 21 and 28, the MDA levels in the skin tissues dropped sharply, and there was no significant difference between the two groups $(P>0.05)$.

During the experimental period, the SOD levels in the skin tissues (Fig. 7B) increased from day 7 to 28 in DW group, whereas they first decreased (from day 7 to 14) and then increased (from day 14 to 28) in the HRW group. On day 7, compared with the SOD levels in the skin tissue in the DW group (12.36 \pm $0.96 \mathrm{U} / \mathrm{mg})$, the same in the HRW group $(22.82 \pm 1.57 \mathrm{U} / \mathrm{mg})$ was significantly higher $(P<0.05)$. On day 
21 , the SOD levels in the DW group $(23.36 \pm 4.04 \mathrm{U} / \mathrm{mg})$ were significantly lower than that in the HRW group $(31.23 \pm 6.13 \mathrm{U} / \mathrm{mg})(P<0.05)$. On day 28 , the SOD levels in the DW group $(24.39 \pm 2.87 \mathrm{U} / \mathrm{mg})$ were significantly lower than that in the HRW group $(33.78 \pm 7.11 \mathrm{U} / \mathrm{mg})(P<0.05)$.

During the experimental period, the gene expression of Nrf-2 in HRW/DW ratio was extremely significantly different between days 7 and 21, and was significantly different on day 14 (Fig. 7C). The gene expression of HO-1 in HRW/DW ratio was extremely significantly different between days 7 and 21 (Fig. 7D). The gene expression of NQO-1 in HRW/DW ratio was extremely significantly difference between days 7 and 21 , and was significantly different on day 14 (Fig. 7E).

\section{Growth Factor Detection}

During the experimental period, the gene expression of VEGF in HRW/DW ratio was extremely significantly different between days 7 and 14 (Fig. 8A). The gene expression of PDGF in HRW/DW ratio was also extremely significantly different between days 7 and 14 (Fig. 8B).

\section{Discussion}

It has long been reported that hydrogen gas can achieve therapeutic effects through anti-oxidation. In 1975, Dole M found that high-pressure hydrogen gas could effectively treat malignant skin tumors [23]. HRW has also shown positive therapeutic effects in many studies [20-22]. When $\mathrm{H} 2$ enters the body and takes part in the reaction, only water is produced, and the excess $\mathrm{H} 2$ is discharged from the body, implying that HRW can be safely used to treat diseases without any side-effects. This is the first study in veterinary-related research to purposefully assess the effects of HRW on simple cutaneous wound healing in dogs. In this study, the positive effect of HRW treatment on skin wound healing in dogs was determined, which was primarily attributed to the its antioxidant potential.

The gold-standard assessment of skin wound healing involves the visual inspection of the wound for surface epithelization and reduction in the skin wound size with time [24]. Recent studies have shown that estrogen might be involved in the wound-healing process at the molecular level [25-27]. Thus, the dogs used in this study were all intact males to avoid differing levels of estrogen. In this established skin wound healing model, we measured the area of skin wounds using Image $\mathrm{J}$ software with the polygon selection tool. The HRW treatment regimen exhibited substantial effects between days 7 and 28; the area of skin wounds was always less than that in the DW group. HRW was found to facilitate the rapid formation of granulation tissue to shorten the healing time than the DW group, which was probably due to the fact that HRW promoted the formation of blood vessels in the early stage of wound healing as well as relatively rapid re-epithelialization. Here, HRW upregulated the expression of antioxidant genes and growth factor genes, which promoted wound healing. Additionally, the wound length in the HRW group was also significantly shorter than that in the DW group (Fig. 4) between days 7 and 14.

Collagen fibers is the main extracellular matrix component, which acts as a structural scaffold in tissues, regulating cell proliferation and migration during skin wound healing [28]. Thus, the content of collagen 
fibers is an important parameter to examine skin wound healing [23]. The HRW group showed that the collagen fibers within the skin wound site were observed to be more regular and higher in content compared to the DW group (from days 7 and 21, Fig. 6). Upregulated gene expression in PDGF stimulated collagen synthesis and the release of collagenase [29] (Fig. 8B). Thus, elevated gene expression of PDGF resulted in more collagen fibers in the HRW group than the DW group. It also explained why collagen fibers increased in skin tissue after treatment with HRW in skin wound healing and was probably one of the major principles involved in HRW-mediated skin wound healing.

During the early stages of skin wound healing, an inflammatory response followed by re-epithelialization of the wound area occurs along with the establishment of granulation tissue accompanied by neovascularization [30]. Among them, neovascularization is indispensable for wound healing and tissue regeneration [31]. Neovascularization provides nutrients and oxygen to the wound, supports keratinocyte migration, transports mesenchymal stem cells to the skin, and then supports wound regeneration [32]. Angiogenesis is a process orchestrated by multiple angiogenic factors, among which VEGF is an essential growth factor regulating the critical steps of angiogenic processes [33]. At the same time, PDGF regulates angiogenesis by recruiting and primes the pericytes [34], promoting skin wound healing. In our experiment, the gene expression of VEGF (Fig. 8A) and PDGF (Fig. 8B) in both groups showed that the HRW group exhibited an enhanced ability for neovascularization at an early stage of skin wound healing (0-7 days), and complied with the results of the number of neovascular in the tissue section (Fig. 5A). Therefore, it was plausible that the effect of HRW on angiogenesis in skin wounds was regulated by increasing the expression of VEGF and PDGF, promoting the formation of granulation tissue. Studies have shown that VEGF increases cartilage formation during the early stages of endochondral bone formation, leading to a significant enhancement in bone formation and bone healing [35]. Fan et al. found that in endometrial repair, VEGF blockade dramatically inhibited re-epithelialization [36]. Elevated VEGF expression could drive re-epithelialization of the skin wound. Therefore, elevated gene expression of VEGF and angiogenesis were the positive signals during the skin wound healing process.

Nrf-2 is a critical regulator of antioxidant response [37], which can avoid the oxidative trauma as well as the development of oxidative stress. MDA is a metabolite of lipid peroxidation of unsaturated fatty acids by oxygen free radicals and indirectly reflects the severity of damage induced by free radicals $[9,28]$. The changes in MDA levels reflect the changes in oxygen-free radical content in the tissues. As a scavenger of free radicals, the SOD activity also reflects the number of free radicals [38]. The antioxidant roles of HRW were central to promoting wound healing. In the present study, we found that HRW activated the Nrf-2 antioxidant pathway and influenced SOD activity and MDA levels in skin tissue of dogs.

The NQO-1 and HO-1 genes are the downstream targets of Nrf-2 and these two genes are regulated by Nrf-2 expression, which is regarded as one of the most important intracellular antioxidant mechanisms [39]. Research has shown that hydrogen molecules can activate the expression of Nrf-2 in lung tissue, thereby promoting the expression of HO-1 and NQO-1 [22]. In our study, it was demonstrated that HRW treatment increased the expression of Nrf-2 (Fig. 7C, from days 7 to 21) and then elevated the expression of its downstream phase II metabolic enzymes HO-1 (Fig. 7D, from days 7 to 21) and NQO-1 (Fig. 7E, from 
days 7 to 21). Nrf-2 can trigger transcription of SOD, reducing the MDA content in the skin tissue [40]. In our experiment, from days 7 to 14, the MDA content in the DW group was significantly higher than that in the HRW group $(P<0.01)$, and the SOD activity in the HRW group was significantly higher than that in the DW group $(P<0.05)$ on days 7,21 , and 28 . As one of the reactive intermediates from oxidation, MDA is a marker of fatty acid oxidation; its elevated levels might lead to impaired and delayed skin wound healing in rats $[41,42]$. Li et al. found that wound lipid peroxidation and enhancement of SOD expression promoted the wound healing of chronic ulcers [43]. In the diabetic model, increased proinflammatory cytokines and oxidative stress in the wound microenvironment resulted in the delay of wound healing [44]. This suggested that the activity of SOD reflected the oxidation levels of skin tissue, and lower oxidation level could avoid oxidative damage, causing faster healing. Ohsawa et al. reported that $2 \%$ hydrogen gas inhaled by animals could scavenge hydroxyl radical $(\mathrm{OH} \cdot)$ and peroxynitrite anion (ONOO-), thereby improving oxidative stress-induced cell injury [45]. SOD can catalyze the dismutation of superoxide anions into oxygen and hydrogen peroxide, and this step also requires the participation of hydrogen. HRW provides more hydrogen for SOD to participate in the scavenging of oxygen radicals; therefore, HRW scavenges the hydroxyl radicals and superoxide radicals by enhancing the SOD vitality, thereby reducing MDA content, promoting skin wound healing.

\section{Conclusion}

We demonstrated that HRW could increase the number of blood vessels in the granulation tissue, accelerating wound epithelization, reducing inflammatory reaction, stimulating the expression of cytokines related to wound healing, shortening wound healing time, thereby accelerating skin wound healing. The dogs showed no discomfort in following the treatment strategy. These results showed that $\mathrm{HRW}$ is a safe and effective veterinary clinical treatment for dog skin wound healing.

\section{Abbreviations}

HRW: Hydrogen-rich water

DW: Distilled water

HRS: Hydrogen-rich saline

MDA: Malondialdehyde

SOD: Superoxide dismutase

\section{Declarations}

\section{Availability of data and materials}


The datasets generated and/or analysed during the current study are available from the corresponding author on request.

\section{Acknowledgments}

The authors would like to thank Dr. Zhong-Wang Liu of the Institutes of Brain Science, Fudan University and Dr. Jia-Feng Ding of Northeast Agricultural University for their suggestions on the article, as well as M.S. Hao Xia of Suzhou University of science and technology for provided support for figure processing.

\section{Funding}

This study was funded by the by the National Natural Science Foundation of China (31402261), "Academic Backbone"Project of Northeast Agricultural University (18XG22).

The funding body did not have any influence over the design, analysis and reporting of the study.

\section{Contributions}

Dong-Dong Qi, Writing original article, performed experiments, data collection; Ting Wang, Performed Analysis, contributed in all experiments; Muhammad Abid Hayat, revising the English of this article; Tao Liu, Contributed in all experiments; Jian-Tao Zhang, Conception and design of the work, revising the article.

\section{Ethics approval and consent to participate}

The Laboratory Animal Welfare and Ethics Committee of Northeast Agricultural University (\#NEAU-201904-0245-13) approved the experimental design and animal surgery procedures in this study. The study was carried out in compliance with the ARRIVE guidelines. All methods were handled in accordance with good animal practices required by the Animal Ethics Procedures and Guidelines of the People's Republic of China.

\section{Consent for publication}

Not applicable.

\section{Competing interests}

The authors declare that they have no competing interests.

The study was carried out in compliance with the ARRIVE guidelines

\section{References}

1. Joanna JK. Novel method for border irregularity assessment in dermoscopic color images. Comput Math Methods Med. 2015; 2015: 496202. 
2. Wang Y, Jing L, Zhao XM, Han JJ, Xia ZL, Qin SC, Wu YP, Sun XJ. Protective effects of hydrogen-rich saline on monocrotaline-induced pulmonary hypertension in a rat model. Respir Res. 2011; 12(1): 26-33.

3. Xu L, Mclennan SV, Lo L, Natfaji A, Bolton T, Liu Y, Twigg SM, Yue DK. Bacterial load predicts healing rate in neuropathic diabetic foot ulcers. Diabetes Care. 2007; 30(2): 378-380.

4. Fu XB, Fang LJ, Li HH, Li XK, Cheng B, Sheng, ZY. Adipose tissue extract enhances skin wound healing. Wound Repair Regener. 2010; 15(4): 540-548.

5. Hage R, Plapler H, Bitar RA. Photodynamic therapy (PDT) to treat a chronic skin wound in a dog. Proc Soc Photo Opt Instrum Eng. 2008; 16: 68450X-1-68450X-6.

6. Toyokawa H, Matsui YY, Uhara J, Tsuchiya H, Teshima S, Nakanishi H, Kwon AH, Azuma Y, Nagaoka T, Ogawa T, Kamiyama Y. Promotive effects of far-infrared ray on full-thickness skin wound healing in rats. Exp Biol Med. 2003; 228(6): 724-729.

7. Yang L, Li DC, Chen SY. Hydrogen water reduces NSE, IL-6, and TNF-a levels in hypoxic-ischemic encephalopathy. Open Med. 2016; 11(1): 399-406.

8. Kajiyama S, Hasegawa G, Asano M, Hosoda H, Fukui M, Nakamura N, Kitawaki J, Imai S, Nakano K, Ohta M, Adachi T, Obayashi H, Yoshikawa T. Supplementation of hydrogen-rich water improves lipid and glucose metabolism in patients with type 2 diabetes or impaired glucose tolerance. Nutr Res. 2008, 28: 137-143.

9. Toru I. Molecular hydrogen: new antioxidant and anti-inflammatory therapy for rheumatoid arthritis and related diseases. Curr Pharm Des. 2013; 19(35): 6375-6381.

10. Kawamura T, Wakabayashi N, Shigemura N, Huang CS, Masutani K, Tanaka Y, Noda K, Peng X, Takahashi T, Billiar TR, Okumura M, Toyoda Y, Kensler TW, Nakao A. Hydrogen gas reduces hyperoxic lung injury via the Nrf2 pathway in vivo. Am J Physiol Lung Cell Mol Physiol. 2013; 304(10): 646656.

11. Watanabe S, Fujita M, Ishihara M, Tachibana, S, Yamamoto Y, Kaji T, Kawauchi T, Kanatani Y. Protective effect of inhalation of hydrogen gas on radiation-induced dermatitis and skin injury in rats. J Radiat Res. 2014; 55(6): 1107-1113.

12. Christian RL, Cassie NL, Sarah R, Marti GD, Cheryl B, Mee MS. Effects of hyperbaric oxygen therapy on uncomplicated incisional and open wound healing in dogs. Veterinary surgery 2018; 47: 827-836.

13. Ohsawa I, Ishikawa M, Takahashi K, Watanabe M, Nishimaki K, Yamagata K, Katsura K, Katayama Y, Asoh S, Ohta S. Hydrogen acts as a therapeutic antioxidant byselectively reducing cytotoxic oxygen radicals. Nat Med. 2007; 13(6): 688-694.

14. Ohta Shigeo. Molecular hydrogen as a preventive and therapeutic medical gas: initiation, development and potential of hydrogen medicine. Pharmacol Ther. 2014; 144(1): 1-11.

15. Niu YH, Nie QR, Dong LP, Zhang JH, Song DM. Hydrogen attenuates allergic inflammation by reversing energy metabolic pathway switch. Sci Rep. 2020; 10: 1962-1974.

16. Masanori K, Michio H, Yoko T, Osamu S. Hydrogen-rich water inhibits glucose and a, $\beta$-dicarbonyl compound-induced reactive oxygen species production in the SHR.Cg-Leprcp/NDmcr rat kidney. Med 
Gas Res. 2012; 2: 18-24.

17. Shin EM, Zhou HY, Guo LY, Kim JA, Lee SH, Merfort I, Kang SS, Kim HS, Kim S, Kim YS. Antiinflammatory effects of glycyrol isolated from Glycyrrhiza uralensis in LPS-stimulated RAW264.7 macrophages. Int Immunopharmacol. 2008; 8(11): 1524-1532.

18. Xu Z, Zhou JM, Cai JM, Zhu Z, Sun XJ, Jiang CL. Anti-inflammation effects of hydrogen saline in LPS activated macrophages and carrageenan induced paw oedema. BioMed Central. 2012; 9(1): 29.

19. Botek M, Krejčí J, McKune AJ, Sládečková B. Hydrogen-rich water supplementation and Up-Hill running performance: Effect of athlete performance level. Int J Sports Physiol Perform. 2020; 10: 14.

20. Song DD, Liu XL, Diao YG Sun YJ, Gao GJ, Zhang TZ, Chen KY, Pei L. Hydrogen-rich solution against myocardial injury and aquaporin expression via the PI3K/Akt signaling pathway during cardiopulmonary bypass in rats. Mol Med. Rep. 2018; 18: 1925-1938.

21. Madigan MC, McEnaney RM, Shukla AJ, Hong, G, Kelley, EE, Tarpey, MM, Gladwin, M, Zuckerbraun, BS, Tzeng, E. Xanthine oxidoreductase function contributes to normal wound healing. Mol Med. 2015; 21(1): 313-322.

22. Naofumi T, Cristina OR, Makoto F, Hiro Ol. Hydrogen-rich water intake accelerates oral palatal wound healing via activation of the Nrf2/antioxidant defense pathways in a rat model. Oxid Med Cell Longevity. 2016; 2016: 5679040.

23. Dole M, Wilson F, and Fife W. Hyperbaric hydrogen therapy: a possible treatment for cancer. Science. 1975; 190(4210): 152-154.

24. Jayachandran M, Rodriguez S, Solis E, Lei JL. Godavarty A. Critical review of noninvasive optical technologies for wound imaging. Adv Wound Care. 2016; 5: 349-359.

25. Ashcroft GS, Greenwell WT, Horan MA, WahI SM, Ferguson MJ. Topical estrogen accelerates cutaneous wound healing in aged humans associated with an altered inflammatory response. AM $J$ Pathol. 1999; 155(4): 1137-1146.

26. Balgobin S, Montoya, TI, Shi HL, Acevedo JF, Keller PW, Riegel M, Wai CY, Word RA, Estrogen alters remodeling of the vaginal wall after surgical injury in guinea pigs. Biol Reprod. 2013; 89(6): 1-10.

27. Horng HC, Chang WH, Yeh CC, Huang BS, Chang CP, Chen YJ, Tsui KH, Wang PH. Estrogen effects on wound healing. Int J Mol Sci. 2017; 18(11): 2325-2338.

28. Soundia A, Hadaya D, Esfandi N, Gkouveris I, Christensen R, Dry SM, Bezouglaia O, Pirih F, Nikitakis N, Aghaloo T, and Tetradis S. Zoledronate impairs socket healing after extraction of teeth with experimental periodontitis. J Dent Res. 2018; 97(3): 312-320.

29. Park U, Kim K. Multiple growth factor delivery for skin tissue engineering application. Biotechnol Bioprocess Eng. 2017; 22(6): 659-670.

30. Clark RA. Biology of dermal wound repair. Dermatol Clin. 1993; 11: 647-666. 
31. Jessica, Ratajczak, Tim, Vangansewinkel, Pascal, Gervois, Greet, Merckx, Petra, Hilkens, Marc, Quirynen, Ivo, Lambrichts, Annelies, Bronckaers. Angiogenic properties of 'leukocyte- and platelet-rich fibrin'. Sci Rep. 2018; 8: 14632.

32. Mazzon E, Esposito E, Paola RD, Muià C, Crisafulli C, Genovese T, Caminiti R, Meli R, Bramanti P, Cuzzocrea S. Effect of tumour necrosis factor-a receptor 1 genetic deletion on carrageenan-induced acute inflammation: a comparison with etanercept. Clin Exp Immunol. 2008; 153: 136-149.

33. Ferrara N, Gerber HP, Lecouter J. The biology of VEGF and its receptors. J Nat Med. 2003; 9(6): 669676.

34. Potente M, Gerhardt H, and Carmeliet P. Basic and therapeutic aspects of angiogenesis.. Cell. 2011; 146(6): 873-887.

35. Pizzicannella J, Gugliandolo A, Orsini T, Fontana A, and Trubiani O. Engineered extracellular vesicles from human periodontal-ligament stem cells increase VEGF/VEGFR2 expression during bone regeneration image_1.TIF image_2.TIF. Front Physiol. 2019; 10: 1-16.

36. Fan XJ, Krieg S, Kuo CJ, Wiegand SJ, Rabinovitch M, Druzin ML, Brenner RM, Giudice LC, Nayak NR.VEGF blockade inhibits angiogenesis and reepithelialization of endometrium. The FASEB Journal. 2008; 22: 3571-3580.

37. Bai T, Lei PX, Zhou H, Liang RP, Sun YL. Sigmareceptor protects against ferroptosis in hepatocellular carcinoma cells. Cell Mol Med. 2019; 23: 1-11.

38. Wang AH, Si ZH, Li XL, Lu L, Pan YL, Liu JZ. FK506 Attenuated pilocarpine-induced epilepsy by reducing inflammation in rats. Front Neurol. 2019; 10: 971-979.

39. Xiao WG, Jia ZH, Zhang QY, Wei C, Wang HT, Wu YL. Inflammation and oxidative stress, rather than hypoxia, are predominant factors promoting angiogenesis in the initial phases of atherosclerosis. Mol Med Rep. 2015; 12: 3315-3322.

40. Huang XS, Chen HP, Yu HH, Yan YF, Liao ZP, Huang QR. Nrf2-dependent upregulation of antioxidative enzymes: a novel pathway for hypoxic preconditioning-mediated delayed cardioprotection. Mol Cell Biochem. 2014; 385(1-2): 33-41.

41. Gupta A, Singh RL, Raghubir R. Antioxidant status during cutaneous wound healing in immunocompromised rats. Recent Res Dev Mol Cell Biochem. 2002; 241(1-2): 1-7.

42. Imene A, Sana B, Massara M, Sahnoun Z, Rebaii T, Attia H, Ennouri M. Antioxidant, antibacterial and in vivo dermal wound healing effects of Opuntia flower extracts. Int J Biol Macromol. 2015; 81: 483490.

43. Li XT, Zhang YK, Kuang HX, Jin FX, Liu DW, Gao MB, Liu Z, Xin XJ. Mitochondrial protection and antiaging activity of Astragalus polysaccharides and their potential mechanism. Int J Mol Sci. 2012 13(2): 1747-1761.

44. Dhall S, Hoffman T, Sathyamoorthy M, Lerch A, Danilkovitch, A. A viable lyopreserved amniotic membrane modulates diabetic wound microenvironment and accelerates wound closure. Adv Wound Care. 2019; 8(8): 355-367. 
45. Eisner MD, Thompson T, Hudson LD, Luce JM, Matthay MA. Efficacy of low tidal volume ventilation in patients with different clinical risk factors for acute lung injury and the acute respiratory distress syndrome. Am J Respir Crit Care Med. 2001; 164(2): 231-236.

\section{Figures}

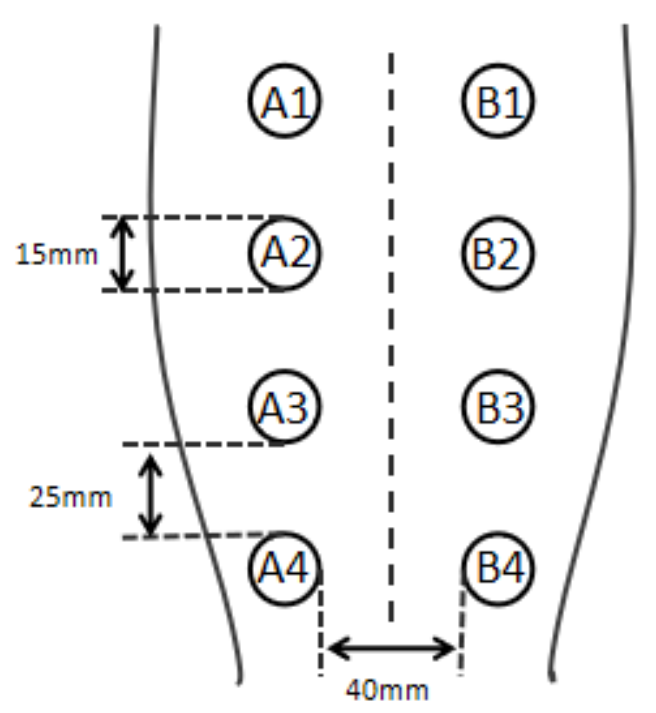

\section{Figure 1}

Sampling Methods: Four circular wounds with a diameter of $15 \mathrm{~mm}$ on each side of the midline of the shaved dorsum. Each wound is $20 \mathrm{~mm}$ from the midline of the dorsum, and the adjacent wounds are 25 $\mathrm{mm}$ from each other. From the head side to the tail side, and from left to right, the wounds are labeled in the following order: $A 1, A 2, A 3, A 4, B 1, B 2, B 3, B 4$. 

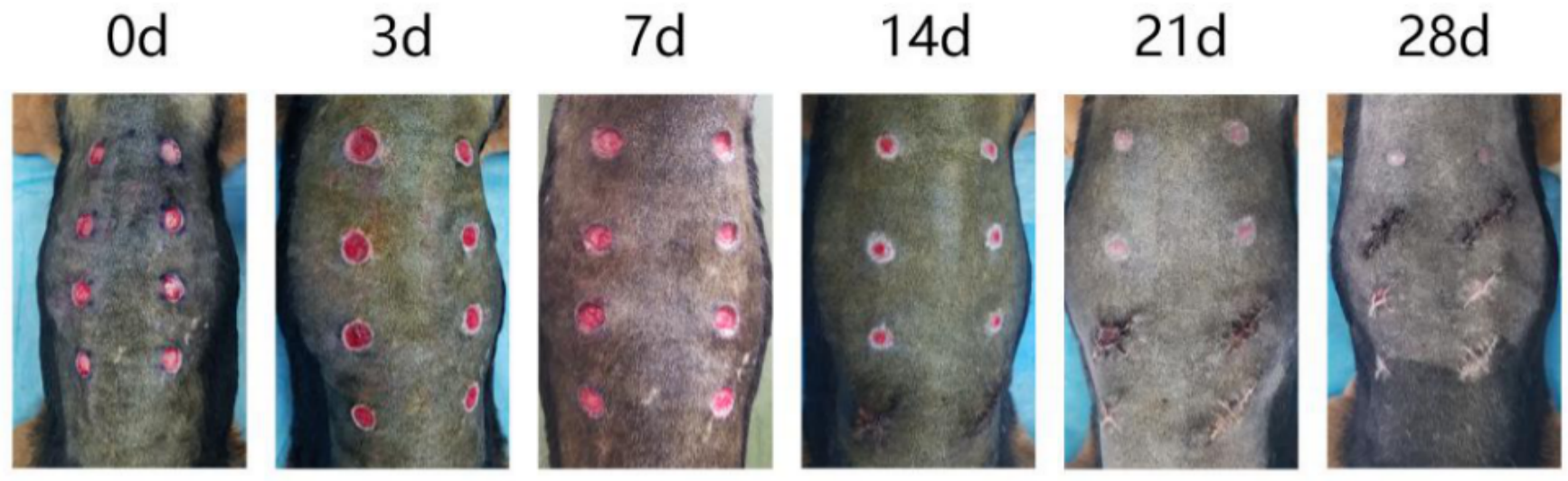

HRW
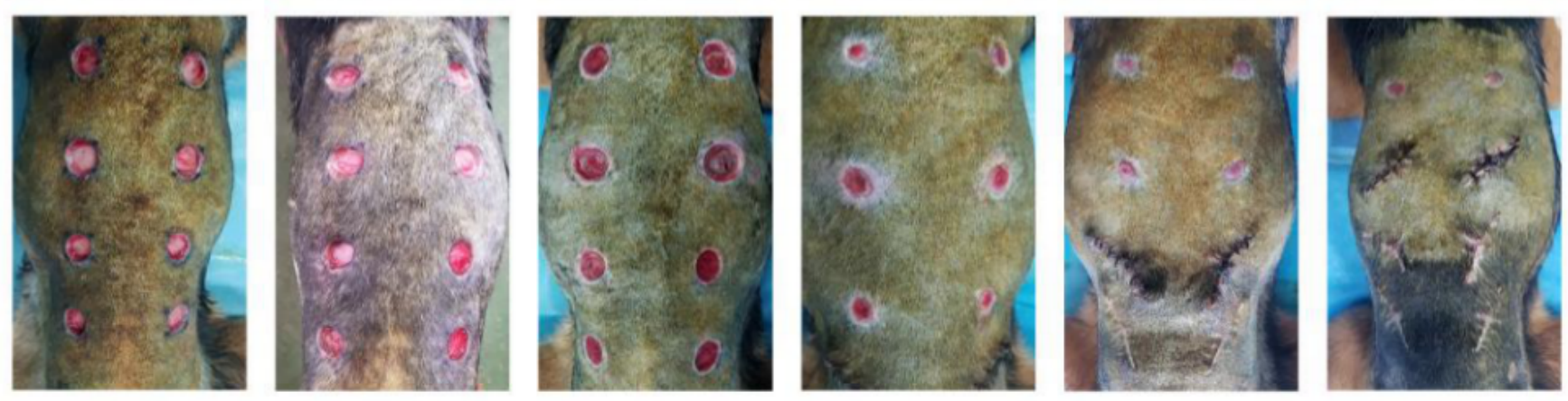

\section{DW}

Figure 2

Wound healing at each time point: (HRW) Images of dogs in the experimental group at days $0,3,7,14,21$, and 28 after modeling; (DW) Images of dogs in the control group at days $0,3,7,14,21$, and 28 after modeling. The same dog was photographed at each time point.
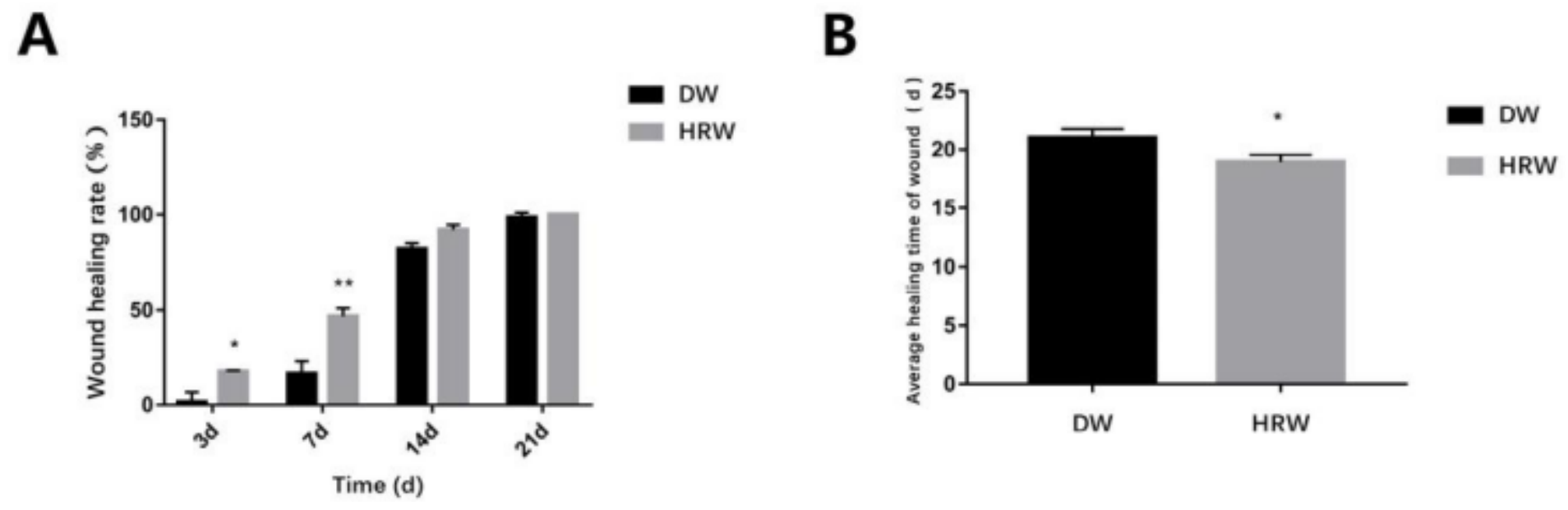

Figure 3

Average wound healing rate and time: (A) The wound healing rate on days $3,7,14$, and 21 after surgical operation between HRW and DW, $* P<0.05,{ }^{*} P<0.01$. (B) After surgical operation until the skin wound was completely covered by the granulation tissue was considered the healing time of wound. Compared with two groups, ${ }^{*} \mathrm{P}<0.05$. 


\section{HRW}

7d

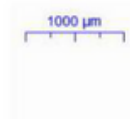

DW
7d

HRW

14d
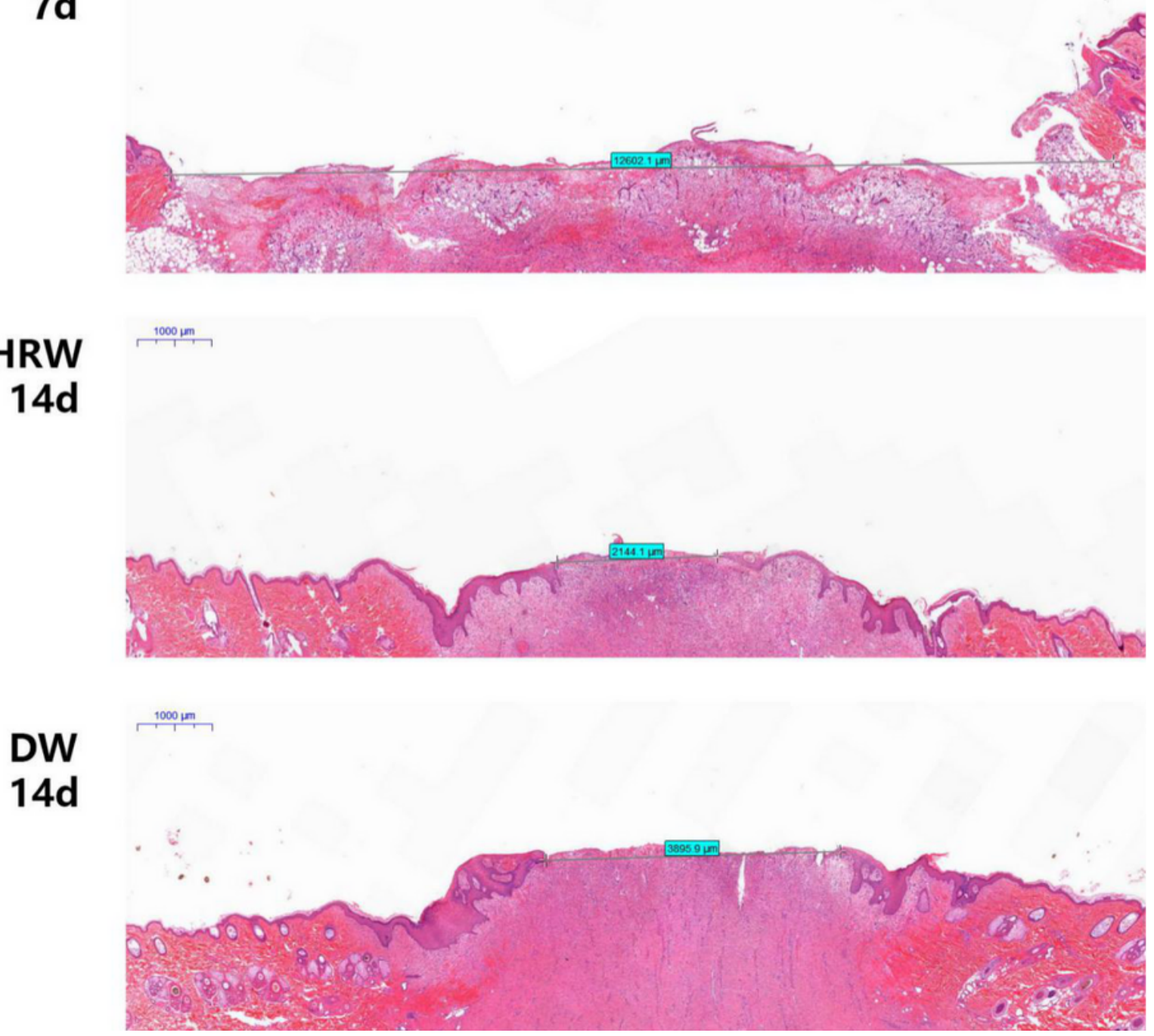

\section{Figure 4}

H\&E section of wound length: H\&E section of the wound in the experimental group and the control group on days 7 and 14 . H\&E staining was used to measure the length of wound of dogs. 
A

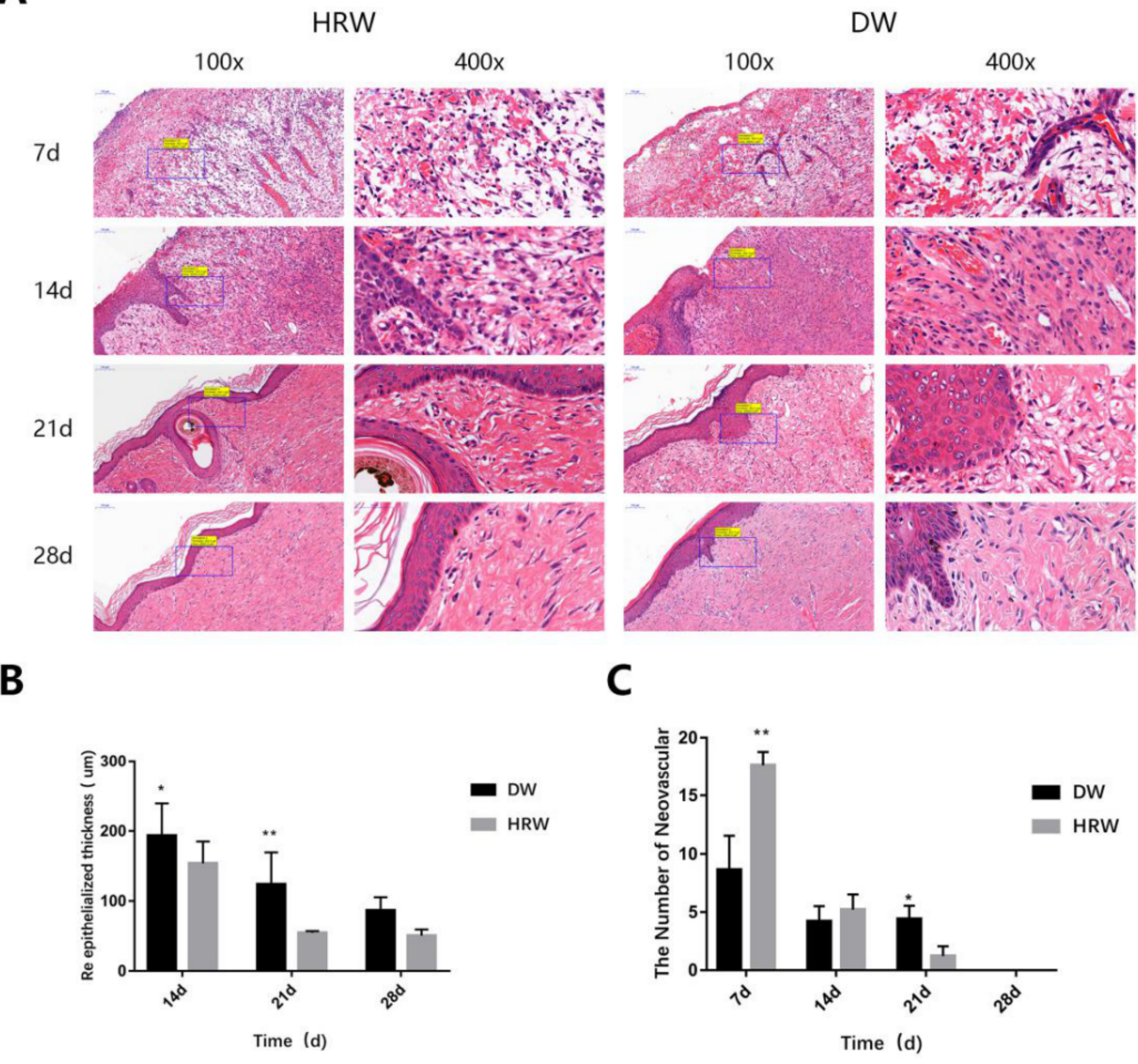

Figure 5

Wound healing assessment of HE section: (A) H\&E section of the skin wound. Angiogenesis, granulation tissue, and re-epithelialization were observed by H\&E staining. (B) Re-epithelialized thickness. H\&E samples were observed, and the epidermal thickness at the wound edge was measured using ImageJ. Reepithelialized thickness in the HRW (153.42 $\pm 31.72 \mu \mathrm{m})$ and DW (193.28 $\pm 45.80 \mu \mathrm{m})$ groups on day 14; $\operatorname{HRW}(54.16 \pm 2.53 \mu \mathrm{m})$ and DW $(123.54 \pm 46.14 \mu \mathrm{m})$ on day $21 ; \mathrm{HRW}(50.12 \pm 8.69 \mu \mathrm{m})$ and DW (86.50 \pm $18.78 \mu \mathrm{m}$ ) on day $28,{ }^{*} \mathrm{P}<0.05,{ }^{*} \mathrm{P}<0.01$. (C) Average number of blood vessels. The number of neovascular structures in skin tissue were assessed by H\&E staining of the paraffin-embedded section. 
The number of neovascular structures in the HRW $(17.60 \pm 1.14)$ and DW $(8.60 \pm 2.97)$ groups on day 7 ; HRW (4.20 \pm 1.41$)$ and DW (5.20 \pm 1.31$)$ on day 14 , HRW (1.20 \pm 0.84$)$ and DW (3.20 \pm 0.81$)$ on day 21 , $\star \mathrm{P}<0.05,{ }^{*} \mathrm{P}<0.01$.

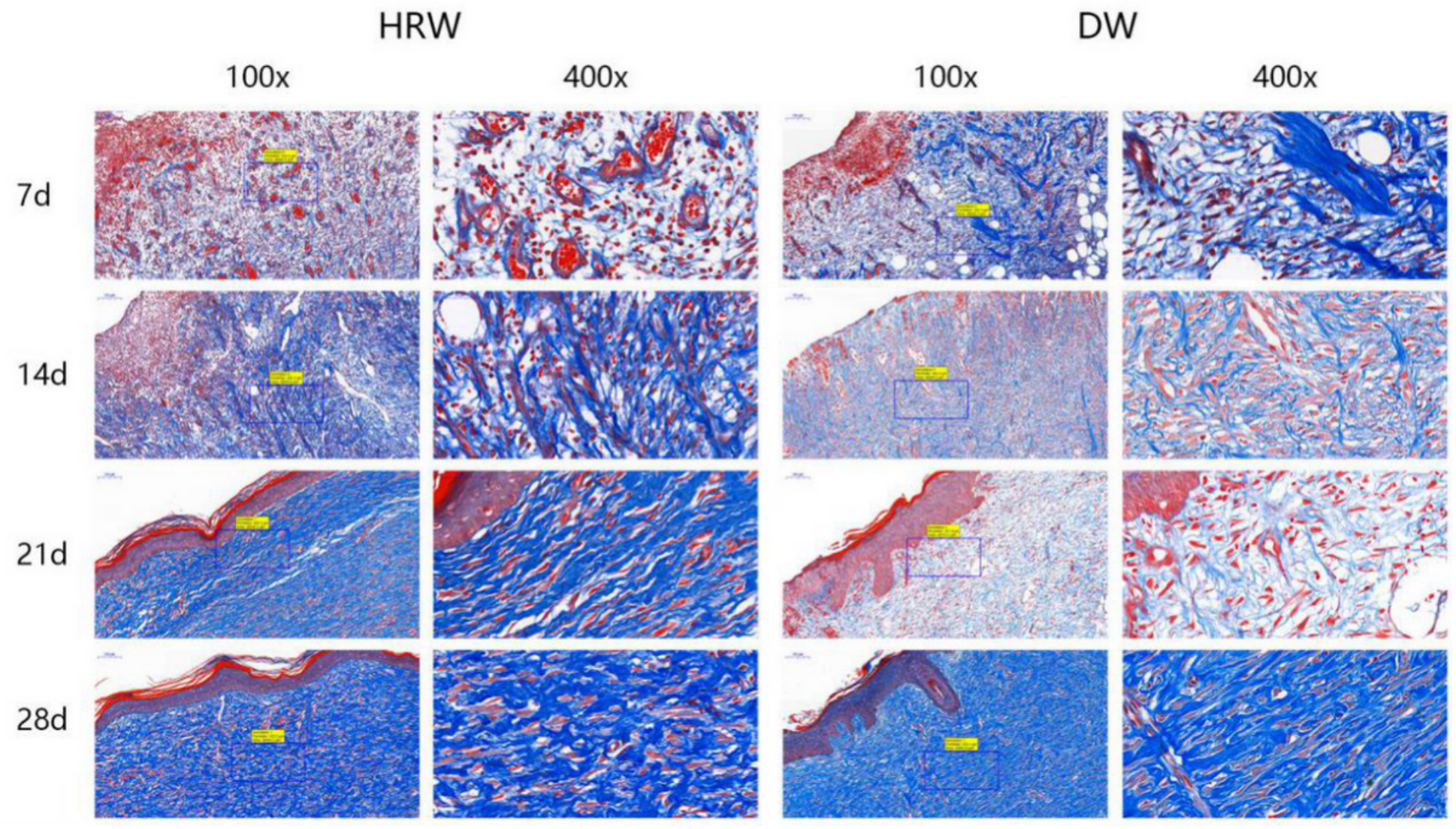

Figure 6

Masson section of wound: Skin wound paraffin sections were subjected to Masson staining. The collagen fibers were stained blue, and the muscle fibers were stained red after Masson's trichrome staining. 
A

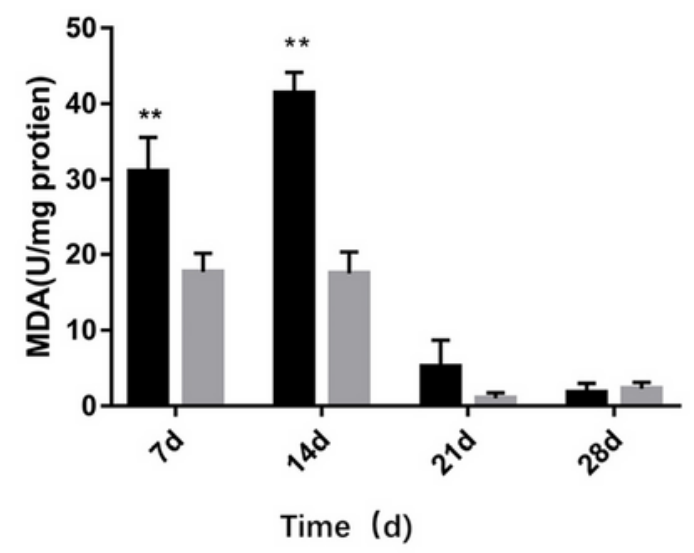

C

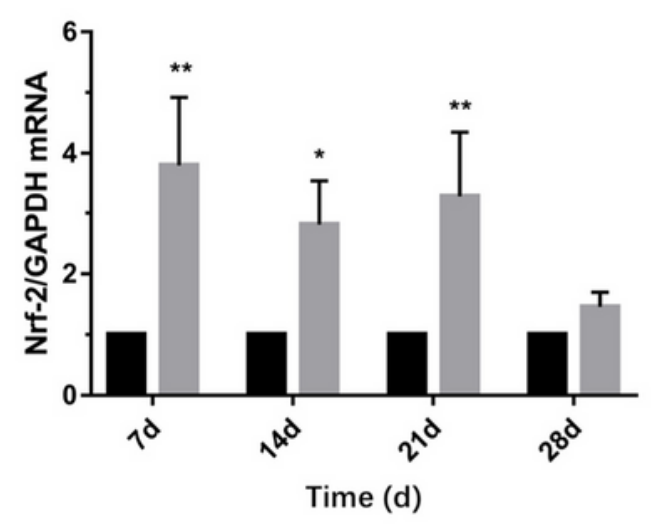

$\mathbf{E}$

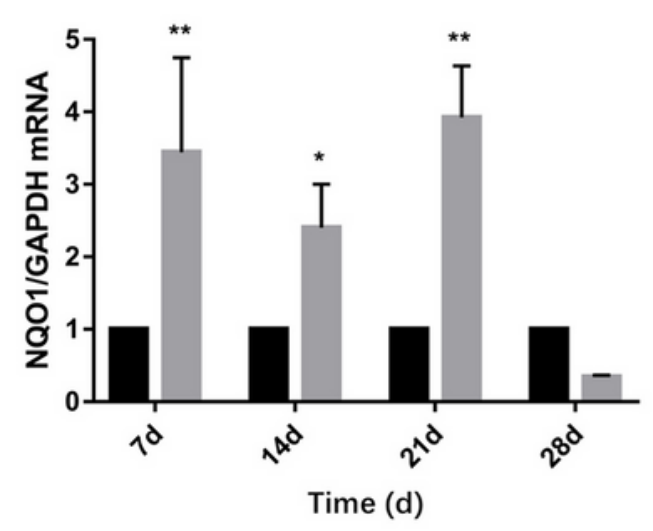

B

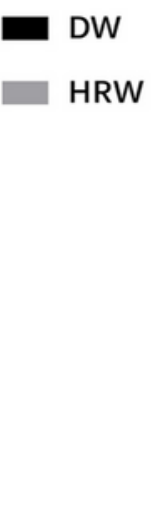

D
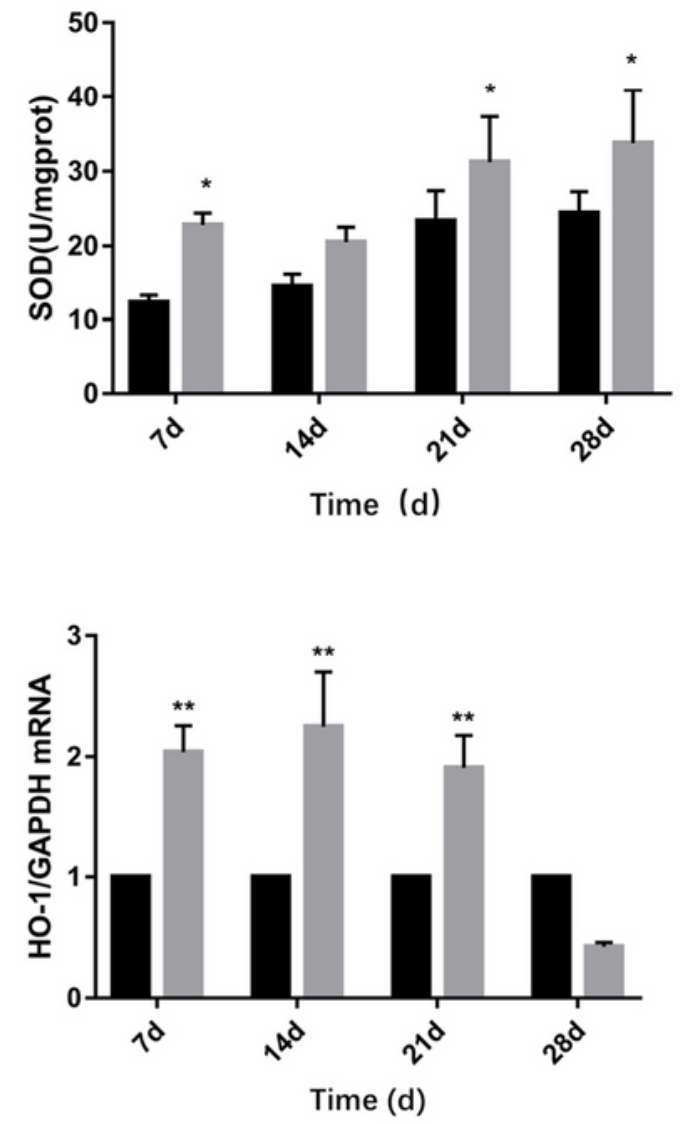

Figure 7

Antioxidant indices: (A) MDA activity of sample was determined using test kits. The DW group had higher MDA levels than the HRW group on days 7 and $14,{ }^{*} P<0.05$, $* * P<0.01$. (B) SOD activity of sample was determined using test kits. The HRW group had higher SOD activity than the DW group on days 7,21 , and $28,{ }^{*} \mathrm{P}<0.05,{ }^{\star} \mathrm{P}<0.01$. (C) Nrf-2 gene content. Relative Nrf-2 mRNA levels were measured by TaqMan real-time PCR. The HRW group had higher mRNA expression than DW group on days 7,14 , and 21 , *P< 
$0.05,{ }^{*} \mathrm{P}<0.01$. (D) HO-1 gene content. Relative HO-1 mRNA levels were measured by TaqMan real-time PCR. The HRW group had higher mRNA levels than the DW group on days 7,14 , and $21,{ }^{\star} * P<0.01$. (E) NQO-1 gene content. Relative NQO-1 mRNA levels were measured by TaqMan real-time PCR. The HRW group had higher NQO-1 gene levels than the DW group on days 7, 14, and $21,{ }^{*} P<0.05,{ }^{*} \mathrm{P}<0.01$.

A

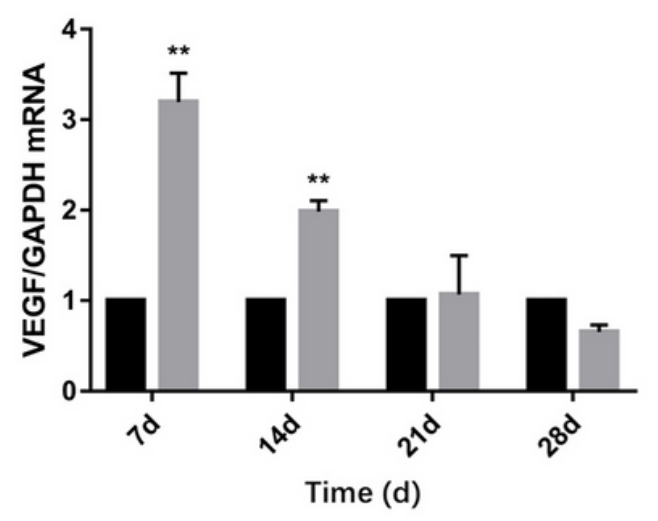

B

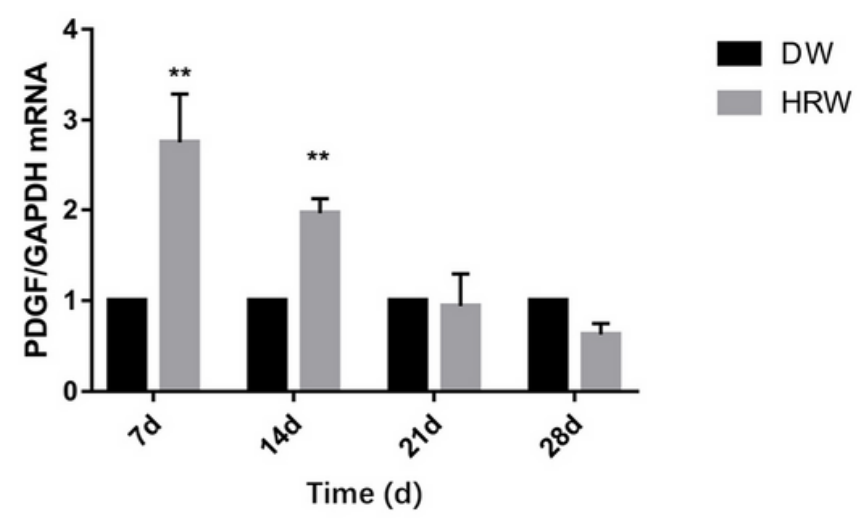

\section{Figure 8}

The gene expression levels of growth factor: (A) VEGF gene content. Relative VEGF mRNA levels were measured by TaqMan real-time PCR. The HRW group had higher mRNA levels than DW group on days 7 and 14, $\star * P<0.01$. (B) PDGF gene content. Relative PDGF mRNA levels were measured by TaqMan realtime PCR. The HRW group had higher mRNA levels than DW group on days 7 and $14, * \star P<0.01$. 